\section{TREATMENT OF FRACTURED RIBS} BY

DENYS J. NEAL SMITH, M.D.

Resident Surgical Officer, Hendon Cottage Hospital ; Surgeon, E.M.S.

It has long been evident that the usual routine method of treating simple fractures of the ribs by extensive strapping of the chest wall is most unsatisfactory. Relief from pain does occur to some extent, but it is never complete, and the strapping often proves uncomfortable, irritating, and sticky, becoming well-nigh intolerable in a few days.

The aim of treatment by this method is to secure immobilization of the chest wall. It is not clear, however, how strapping is expected to effect such immobilization. For one thing, the skin moves freely over the ribs, and strapping soon stretches, while the very mechanics of rib movement make it virtually impossible to immobilize one side of the chest only, as is usually attempted. It must be remembered that the muscles of normal respiration are functionally of the bilateral kind, and, in this connexion, Böhler (1935) points out the necessity of completely encircling the chest with strapping. As, however, ordinary individuals are not trained to breathe with the diaphragm alone (even though this may be theoretically possible), it seems clear that immobilization of a part or the whole of the chest wall is an ideal impossible of attainment.

A further point of vital importance, and one which is often overlooked, is that immobilization of the chest wall will result in insufficient aeration of the underlying lung. This may not be a very serious matter in a young person, but in the elderly it may predispose to a hypostatic pneumonia. These patients are of necessity confined to bed; the pain of the fracture stops them from moving about, and congestion at the bases may occur, while, in addition, sufferers from chronic bronchitis are unable to cough (in spite of strapping), and retained secretions may be an added factor adversely affecting the lung. Most patients will say. that strapping helps them a great deal when first applied, but sharp pain still occurs if deep breathing, coughing, or a sudden movement be attempted.

\section{Pain the Chief Disability}

It is clear, therefore, that the chief disability is pain, and were there no pain at the site of a fractured rib none of the secondary effects would arise and, indeed, the fracture would be of no consequence (such complications as compound fractures, injuries to the lung, and haemothorax being for the moment excluded).

It has long been accepted that the severe pain of fractured ribs is due to the broken ends rubbing together. It is questionable whether this is altogether true. The ribs are very firmly held between thick powerful muscular layers, and the broken ends must be, to each other, relatively immobile. In other words, when a rib moves it moves as a whole, even if it is in two or more parts, swinging up and outwards rather like a bucket-handle. Watson-Jones (1941) has pointed out that any movement between the bone ends would result in non-union, but this is unheard of in the ribs. This does not imply that there is no tendency for movement to occur, and it is probable that pain arises as a result of the muscles resisting such movement.

In endeavouring to explain the origin of the pain it must also be remembered that the maximum pain, which is of a short sharp stabbing type, takes place under two conditions. One is full inspiration (hence, in a suspected case, the clinical test of asking the patient to breathe deeply) and the other is coughing. In full inspiration the ribs are raised and swung outwards and maximum expansion is occurring. In this position the tendency would obviously be for the bone ends to separate, and, conversely, in expiration they should be forced closer together. If, therefore, the pain were really caused by the bone ends rubbing together, it would be expected to occur not in inspiration but in expiration. It is well known, too, that in other parts of the body relief of pain occurs when the broken ends of a bone separate (e.g., by skeletal traction), and it does not appear that separation of the bone ends alone explains the pain at full inspiration. In coughing, too, the attempted expiration against the resistance of the glottis should also, on mechanical principles, tend to cause separation of the bone ends, probably in this case opening the angle between the fragments as well.

If the pain of a fractured rib be carefully analysed it will be found to consist of a combination of two different pains. One is a constantly present dull ache, not localized exactly to the fracture but extending upwards and downwards two or three spaces and backwards and forwards some distance. The second pain is the sharp stabbing pain already mentioned, produced by deep breathing and coughing. The intensity of the latter pain is such that the former is generally disregarded but is noticed if the latter be abolished.

The dull ache is probably due to the bruising of the tissues (including the bone) which occurs in a wide area around any injured rib, and the sharp pain may possibly be caused by muscular spasm, occurring whenever there is a tendency to movement, particularly separation, of the fragments. Such spasm may be the result of an intense effort on the part of muscle fibres around the fracture to prevent movement between the ends, or it may be due to the over-stretching of a group of muscular fibres attached to one of the fragments. It should be noted that only the tendency towards movement of the bone ends need be present: actual movement is prevented by intense local activity of the muscles, and it is this intense effort that produces the pain.

There is also the possibility of involvement of the intercostal nerve. It is unlikely to be caught between the bone ends, but it might become involved or pressed on by a localized haematoma. Such pressure is likely to be most pronounced in inspiration, when muscle activity in the locality would be at its highest. Nerve involvement may explain the radiation of the pain forwards and backwards in the same space.

The pleura must not be forgotten as a possible painproducer. It is in close relation to the periosteum on the inner side of the rib, and must be bruised, if not torn, and have a haematoma superficial to it.

\section{Treatment}

It was Zoppi, in 1936, who first recommended the injection of procaine at the site of the fracture in a damaged rib. He pointed out that while strapping helped a great deal, morphine often had to be given as well to relieve pain. This produced the most undesirable effect of depressing respiration, with its attendant risks. Zoppi injected procaine at the site of the fracture, procuring immediate relief of pain, and the patient's ability to cough, move, and breathe deeply returned at once. Curiously enough Zoppi's idea was not taken up, but other writers had previously recorded the alcohol injection of the intercostal nerves posteriorly in order to anaesthetize a wider area for serious injury to the chest wall. Labat (1928) described intervertebral block, using procaine and alcohol ; and Latteri (1933) described the alcoholization of intercostal nerves in therapy. Grieco, in 1937, and Rovenstine and Byrd, in 1939, were still using intercostal block with alcohol. They 
were, however, employing the method not for simple fractures but for serious injury to the chest wall, involving several ribs. Zoppi recommended that the injection should be repeated daily, if necessary, to provide continuous relief from pain.

In determining to try the method, I felt that the disadvantage of frequent injections could be overcome by injecting an oily local anaesthetic solution-e.g., percaine in oil, proctocaine, etc. Although such a foreign body as oil might prevent bony union, yet in the ribs this would hardly matter.

Unfortunately, at the time the first case presented itself it was impossible (owing to war conditions) to obtain any oily local anaesthetic solution, and all that could be done was to inject about $7 \mathrm{c.cm}$. of percaine $(1$ in 1,000$)$ in and around the fracture, which was in the sixth rib in the midaxillary line. The sharp stabbing pain disappeared at once, thus confirming that it has its origin at the fracture, but the patient was left with the less localized dull ache. What, however, was really remarkable was that the sharp pain did not return although the anaesthetic action of the percaine could hardly have lasted more than twelve to twentyfour hours. The dull ache, however, persisted for about ten days. The patient was kept in hospital for a week after the injection and then sent home; he was back at work in three weeks.

The second case was that of an elderly woman, a sufferer for years from chronic bronchitis, who had had the fifth and sixth ribs on the left side injured in a car accident. Immediate relief of pain occurred after injection of percaine: she was able to cough freely, and this no doubt saved her from serious lung complications. She, too, was left with what she described as a "bruised" feeling all over one side of the chest, but the sharp stabbing pain never returned.

It seemed almost impossible that relief of pain could be so lasting after only one injection of a non-persistent local anaesthetic, and scepticism was felt until the same thing happened in other cases. Altogether I have dealt with about a dozen cases of simple fractured ribs in this manner, and all have responded in exactly the same way except that in three cases the injection had to be repeated once two or three days later and one case had to have three injections spread over a week; but it was never necessary to resort to an oily solution. It is to be remembered, however, that this is always available should the other method fail to provide continuous relief.

In treating fractured ribs by this method care must be taken not to overlook injury to other structures or the development of a pleural effusion or haemothorax. The latter occurred once in the series of cases under review, and was aspirated at the same time the injection was performed. More serious injury does not exclude local injection, for it must be remembered that the original use was for multiple rib fractures.

From the relief of pain by one injection at the site of injury it appears that some form of permanent change must take place. It is probably the relief of spasm, allowing the fractured surfaces to resume normal relations-perhaps the breaking of some form of vicious circle, as in Leriche's theory of sprains. Certainly it seems difficult of any other explanation.

\section{Technique of Injection}

A few remarks on the technique of the injection may be of value. A 10-c.cm. syringe is required, and two needles are useful although not essential: a fine one for making the preliminary skin wheal, and a larger one of the intramuscular type for the injection of the fracture region itself. The solution used in all the cases was a 1 in 1,000 percaine solution combined with adrenaline. Percaine has proved to be definitely superior to other local anaesthetics in my hands, particularly in fracture work. It was therefore used in these cases, but of course there is no reason why procaine or a similar drug should not be tried.

The site of the fracture is located roughly by the point of maximum tenderness along the affected rib or ribs. Too much reliance should not be placed on radiographs. In many cases they will not show a fractured rib, even when such a fracture is clinically present and can be felt. I have seen two such cases in which no definite fracture could be observed on the radiograph ; but clear clinical evidence was present, and this was confirmed by feeling the fracture with the injecting needle. A haematoma entered by the needle does not indicate absolutely that there is a fracture, although such a haematoma is constantly present with a fracture. Often the injecting needle can clearly detect the fracture.

The site of injection having been chosen, the skin is cleaned and a little iodine applied. Scrupulous care must be taken with the preparation of syringes and needles, which should be boiled, and the anaesthetic solution must be taken from freshly opened ampoules. The danger, particularly in fracture work, of the use of the rubbercapped phial or bottle for solutions does not appear to be sufficiently realized.

A preliminary skin wheal is raised just in front of the fracture with the fine needle, and the larger needle pushed through the overlying tissues in a direction perpendicular to the skin. The needle is then inclined upwards and backwards and slid along the outer surface of the rib. (The obliquity of the ribs is surprising, and the needle must be directed upwards at quite a sharp angle.) Most fractures will be detected in this manner. When the needle is at the fracture site it may be found to have entered a haematoma (as evidenced by withdrawing the plunger), but as it is rarely possible to get the needle between the fractured ends, very often the haematoma will be missed. When the surgeon is satisfied that the needle point is on or in the fracture, about $5 \mathrm{c} . \mathrm{cm}$. of percaine should be injected. The remaining $5 \mathrm{c.cm}$. should be placed around the rib at the site of the fracture. After the needle is withdrawn a small dressing of collodion should be applied to the puncture. Relief of pain will be found to be instantaneous if the injection has been properly performed.

\section{Summary}

A method of treating fractured ribs by a local anaesthetic is described which appears to be an improvement on the old method, and avoids some of its complications. Results of using it are given.

The disadvantages and failure of the old method of treating fractured ribs by strapping are mentioned.

The dangers and difficulty of immobilization of the chest wall are discussed, and the fact that the underlying disability is pain is pointed out.

The origin of pain in fractured ribs is discussed.

My thanks are due to Mr. N. C. Lake, surgeon to Hendon Cottage Hospital, for help and criticism in connexion with the use of this method of treatment on patients in this hospital.

$$
\text { REFERENCES }
$$

Böhler, L. (1935) The Treatment of Fractures, p. 150, Bristol.

Böhler, L. (1935). The Treatment of

Grieco, Fi (1928). Regional Anaesthesia, 2nd. ed., Philadelphia.

Labat, G. (1928). Regional Anaesthesia, 21, 249.

Latteri, S. (1933). Riv. San. Siciliana, 21, 249.

Watson-Jones, R.'(1941). Fractures and Other Bone and Joint Injuries, p. 17, atson-Jones,

Zoppi, B. (1936). Gior. Veneto Sc. Med., 10, 666.

The Commonwealth Fund, New York, has made a grant of $\$ 50,000$ to the American Bureau for Medical Aid to China for the support of the Emergency Medical Service training in Kweiyang, China. 